
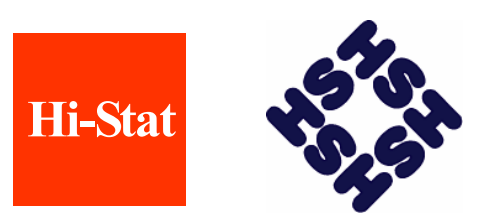

Discussion Paper Series

No.126

\title{
A Test of Cointegration Rank Based on Principal \\ Component Analysis
}

\section{Hiroaki Chigira}

November 2005,

revised January 2006

Hitotsubashi University Research Unit for Statistical Analysis in Social Sciences A 21st-Century COE Program

Institute of Economic Research Hitotsubashi University Kunitachi, Tokyo, 186-8603 Japan http://hi-stat.ier.hit-u.ac.jp/ 


\title{
A Test of Cointegration Rank Based on Principal
}

\author{
Component Analysis
}

\author{
Hiroaki Chigira* \\ Department of Economics, Hitotsubashi University, 2-1 Naka, Kunitachi, Tokyo, 186-8601, \\ Japan
}

November, 2005

Revised, January 2006

\begin{abstract}
This paper considers a test of the rank of cointegration. The test is based on the fact that in an $m$-variate system the $m-r$ th principal component is $I(1)$ under the null of $r$ cointegration rank but $I(0)$ under the alternative of $r+1$ cointegration rank. Exploiting this fact, we construct a cointegration rank test that is less restrictive than Johansen's tests, easy to calculate, and independent of the dimension of the process. Monte Carlo simulations indicate that the proposed test outperforms Johansen's tests, even in the case of a model that satisfies the assumptions required for Johansen's tests and when the sample size is small.
\end{abstract}

Keywords: Cointegration test; Unit roots

JEL classification: C12; C22; C32

\footnotetext{
*E-mail address: hchigira@mercury.ne.jp.
} 


\section{INTRODUCTION}

This paper considers a test for the rank of cointegration. The methodology for testing for cointegration has made great strides in the last few decades and a number of tests to determine the rank of cointegration have been developed. The motivation for this paper is to propose an alternative test for cointegration that tries to deal with some of the problems that arise from a practitioner's point of view.

The proposed test attempts to address two problems in particular. The first is the complexity involved in calculating some test statistics. A commonly used cointegration test is that proposed by Johansen $(1988,1991)$, which is typically included in computer packages to help researchers handle the test. However, researchers stepping outside the circle of Johansen's framework face the tedious challenge of computing some test statistics. And while Johansen's procedure requires that the time series follow an autoregressive process and the disturbances be independent and identically normally distributed, researchers may want to relax these assumption.

The second problem is that the critical values of most cointegration tests depend on the dimension of the time series, $m$, and the cointegration rank under the null hypothesis, $r$. Because critical values of existing cointegration tests are typically not available in series with ten or more variables, it is difficult to analyze a large cointegrated system such as, say, a system consisting of the stock prices of 20 companies.

We therefore believe that it is necessary to explore alternative tests for cointegration that relax the assumptions of Johansen's tests, are easy to calculate and are independent of the dimension of the series and the cointegration rank under the null hypothesis. We propose such a test in this paper, resorting to the fact that in an $m$-variate system cointegrated with rank $r$, the largest $m-r$ principal components are $I(1)$ and the rest of the $r$ principal components are $I(0)$.

Some tests that exploit this property of principal components have already been proposed. The first to apply the principal component method to the analysis of cointegration were Stock and Watson (1988), who did so in the context of Common Trends. Another testing procedure was proposed by Harris (1997), who examined the degree of integration 
of the $r$ smallest principal components using a test statistic based on the one suggested by Kwiatkowski et al. (1992). Harris's test can be applied without specifying the short-run dynamics and the distribution of the error term. However, the critical values depend on $m$ and $r$. One of the interesting features of this test is that it considers the null hypothesis of $r$ cointegration rank against an alternative of $r-1$ cointegration rank. This testing problem is the opposite of Johansen's tests.

Another test for cointegration based on principal component analyses was developed by Snell (1999), which also tests the null of $r$ against the alternative of $r-1$. The test statistic always follows an asymptotic $\chi^{2}$ distribution with a degree of freedom one under the null. That is, this test is independent of $m$ and $r$. However, this test is restrictive in that the short-run dynamics of the series should have finite order autoregressive representation and the order must be estimated.

There are ways other than resorting to the principal component method in order to relax the assumptions of Johansen's tests (see Hubrich, Leutkepohl and Saikkonen 2001 for an excellent survey). The tests developed by Lucas (1997, 1998), for example, are derived from pseudo-likelihood functions which include the Gaussian likelihood as a special case, meaning that he relaxed the Gaussian assumptions. However Lucas's tests still depend on a VAR representation of the process and the dimension of the process. A number of other tests have been developed. Breitung (2002), for instance, proposed a variance ratio test which is similar to the test proposed by Kwiatkowski et al. (1992). Although the specification of the short-run dynamics and distribution of the disturbance are assumed to be unknown in Breitung's setup, the critical values of this test depend on the dimension of the process. Another example is Quintos (1998), who constructed tests which are asymptotically $\chi^{2}$ distributed based on the fully modified estimation approach of Phillips (1995). But although Quintos's tests are virtually independent of the dimension of the process, some assumptions make the model class more restrictive than in Johansen' framework. In sum, there seems to be no test which allows both the relaxation of model restrictions and independence from the dimension of the process.

The idea underlying our testing procedure which addresses both these issues is as follows. Consider the null hypothesis of $r$ cointegration rank against the alternative of 
$r+1$ cointegration rank. Then the $m-r$ th principal component is $I(1)$ under the null but $I(0)$ under the alternative. Therefore, testing the null of $I(1)$ against the alternative of $I(0)$ for the $m-r$ th principal component is equivalent to testing the null of $r$ cointegration rank against the alternative of $r+1$. One can then use a unit root test, such as the Phillips and Perron (hereafter, PP) type unit root test (Phillips (1987) and Phillips and Perron (1988)), to determine the degree of integration of the principal component.

The above testing procedure has the desired properties. It is less restrictive than Johansen's procedure because neither establishing the fact that the $m-r$ principal components are $I(1)$ and the $r$ principal components are $I(0)$ nor the PP test require the autoregressive representation of the series and the distribution of the error term. It is quite easy to apply a commonly-used unit root test such as the PP test to the principal component. A unit root test is obviously independent of $m$ and $r$. In addition, one can apply the idea of this test to flip the hypothesis, i.e., the null of $r$ against $r-1$. In this case, the $m-r$ th principal component is $I(0)$ under the null but $I(1)$ under the alternative. Thus, implementing a unit root test that considers the null of $I(0)$ to the $(m-r+1)$ th principal component leads to a test of the null of $r$ against $r-1$.

The following section introduces the new cointegration test, while Section 3 presents simulation results comparing the new test to Johansen's tests. Section 4 summarizes.

\section{THE TEST FOR COINTEGRATION}

Consider an $m$-variate process that can be written in two components:

$$
y_{t}=\mu+\delta t+x_{t}
$$

where $\mu$ and $\delta t$ are the deterministic components and $x_{t}$ is the stochastic component.

We define $I(1)$ process without specifying a particular model.

Assumption 1. A time series $v_{t}$ is $I(1)$, if

$$
v_{t}=O_{p}(\sqrt{t})
$$


Assumption 2. Let $x_{t}$ in $(1)$ be $I(1)$ and satisfy the following equation:

$$
\begin{aligned}
& \beta^{\prime} x_{t}=O_{p}(1), \\
& \beta_{\perp}^{\prime} x_{t} \sim I(1),
\end{aligned}
$$

where $\beta$ is an $(m \times r)$ matrix of $r$ cointegrating vectors with rank $r, \beta_{\perp}$ is of full rank and dimension $m \times(m-r)$ such that $\beta^{\prime} \beta_{\perp}=0$. This assumption means that (1) is cointegrated. However, we do not assume stationarity of $\beta^{\prime} x_{t}$. Instead, we assume a stochastic order of $\beta^{\prime} x_{t}$.

\section{Assumption 3.}

$$
\begin{aligned}
& \beta^{\prime} S_{\bar{x} \bar{x}} \beta \stackrel{p}{\rightarrow} \text { positive definite matrix, } \\
& \beta_{\perp}^{\prime} S_{\bar{x} \bar{x}} \beta_{\perp}=O_{p}(T), \\
& \beta_{\perp}^{\prime} S_{\bar{x} \bar{x}} \beta=O_{p}(1),
\end{aligned}
$$

where $S_{\bar{x} \bar{x}}=\frac{1}{T} \sum_{t=1}^{T} \bar{x}_{t} \bar{x}_{t}^{\prime}$ with $\bar{x}_{t}$ is the series after removing the mean value or linear trend from $y_{t}$.

Under these assumptions we state the following propositions.

Proposition 1 (Harris (1997) and Snell (1999)). Let $B$ be an $m \times m$ matrix of eigenvectors such that

$$
\begin{aligned}
& S_{\bar{x} \bar{x}}=B \Lambda B^{\prime}, \\
& \Lambda=\left[\begin{array}{cccc}
\lambda_{1} & & 0 & \\
& \lambda_{2} & & \\
& & \ddots & \\
& 0 & & \lambda_{m}
\end{array}\right], \lambda_{1} \geq \lambda_{2} \geq \cdots \geq \lambda_{m} .
\end{aligned}
$$

Let the $m \times(m-r)$ matrix of eigenvectors corresponding to the largest $m-r$ eigenvalues and the $m \times r$ matrix of eigenvectors corresponding to the smallest $r$ eigenvalues be $B_{(m-r)}$ and $B_{(r)}$, respectively. Then

$$
\begin{aligned}
& B_{(m-r)}=\beta_{\perp}\left(\beta_{\perp}^{\prime} \beta_{\perp}\right)^{-1} \beta_{\perp}^{\prime} B_{(m-r)}+O_{p}\left(T^{-1}\right), \\
& B_{(r)}=\beta\left(\beta^{\prime} \beta\right)^{-1} \beta^{\prime} B_{(r)}+O_{p}\left(T^{-1}\right) .
\end{aligned}
$$


Proof. See Harris (1997) and Snell (1999).

Remark 1. Harris (1997) and Snell (1999) state and prove this proposition. However, Harris (1997) assumes that $\beta^{\prime} x_{t}$ and $\beta_{\perp}^{\prime}(1-L) x_{t}$ are stationary and satisfy regularity conditions and Snell (1999) assumes that $\beta^{\prime} x_{t}$ follows the $\operatorname{AR}(p)$ process and $\beta_{\perp}^{\prime}(1-L) x_{t}$ follow the $\mathrm{MA}(\infty)$ process. It should be noted that Proposition 1 can be proved based on Assumptions 2 and 3, which are less restrictive than Harris's (1997) and Snell's (1999) assumptions. The proof, however, is the same as that employed by Harris and Snell and we omit it here.

Remark 2. These results mean that $B_{(m-r)}$ and $B_{(r)}$ estimate $\beta_{\perp}$ and $\beta$ in a sense. Harris $(1997,533)$ explains the relationship between $B_{(r)}$ and $\beta$ as follows: "we are estimating a basis for the space spanned by $\beta$, and not $\beta$ itself'. Snell (1999) shows that $B_{(r)} \stackrel{p}{\rightarrow} \beta R$, where $R$ is a $r \times r$ constant matrix of full rank. We note that $B_{(m-r)} \stackrel{p}{\rightarrow} \beta_{\perp} Q$, where $Q$ is a $(m-r) \times(m-r)$ constant matrix of full rank, can be proved. Its proof, however, is almost the same as that to show $B_{(r)} \stackrel{p}{\rightarrow} \beta R$ and we therefore omit it here.

Proposition 2. Asymptotically, the principal components of a series $\bar{x}_{t}$ have the following properties:

i) $B_{(m-r)}^{\prime} \bar{x}_{t} \sim I(1)$,

ii) $B_{(r)}^{\prime} \bar{x}_{t}=O_{p}(1)$.

Proof: See Appendix.

Remark 3. Snell (1999) provides roughly the same result with assumptions that are a little more restrictive. He assume that $\beta^{\prime} x_{t}$ follows the $\operatorname{AR}(p)$ process and $\beta_{\perp}^{\prime}(1-L) x_{t}$ follows the $\mathrm{MA}(\infty)$ process.

Using the above propositions, we may construct the procedure to decide the cointegra- 
tion rank sequentially. For example, we consider the following testing problem:

$H_{0}$ : the $m-r$ th principal component is $I(1)$

versus

$H_{1}:$ the $m-r$ th principal component is $I(0)$

to test

$H_{0}:$ the cointegration rank is $r$

versus

$H_{1}$ : the cointegration rank is $r+1$.

To determine the cointegration rank we first test the null of $r=0$, that is to say, we implement a unit root test that considers the null of $I(1)$ for the $m$ th principal component. If the null hypothesis is accepted, the cointegration rank is decided to be zero. Otherwise, we then test the hypothesis of $r=1$ or the degree of integration of the $m-1$ th principal component. We sequentially continue to test the null of $I(1)$ for the $m-r$ th principal component as long as the null hypothesis is rejected. When the null of $r=m-1$ is rejected, the cointegration rank is determined to be $m$.

However, it is important to be careful when testing the degree of integration of a principal component. If one applies the PP test to a principal component, it is necessary to impose the assumptions, which is required to apply the PP test in an appropriate manner, on the principal component in addition to the assumptions required to prove the above proposition. Because the PP test does not require normally distributed errors nor AR representation, we proceed by relaxing the assumptions required for Johansen's procedure. However, the assumptions for the PP test of course need to hold. Because the PP test is included in many computer packages, we recommend applying the PP test to the principal component. If one wants to relax the assumptions on the principal component further, one possible way is to use Breitung's (2002) unit root test, which 
is based on less restrictive assumptions than Phillips and Perron's (1988). Explicitly, to exploit Breitung's (2002), we only assume that under the null hypothesis of $I(1)$ a principal component satisfies the functional central limit theorem.

The testing procedure proposed here conforms with the aims stated in the introduction. To prove our proposition, we do not impose the assumption of a normal distribution or a restrictive representation such as autoregression on any variables. Applying an appropriate unit root test that does not require normally distributed errors and autoregressive specification, the test succeeds in the relaxation of the assumptions of Johansen's tests. Once $B^{\prime} y_{t}$ is calculated, it is easy to implement a unit root test. The testing procedure is obviously independent of the dimension of the time series. To test the cointegration rank for 50-variate time series, one runs through a unit root test. In addition, it is easy to flip the hypothesis. Specifically, we consider the following testing problem:

$H_{0}:$ the $(m-r+1)$ th principal component is $I(0)$

versus

$H_{1}:$ the $(m-r+1)$ th principal component is $I(1)$

to test

$H_{0}:$ the cointegration rank is $r$

versus

$H_{1}$ : the cointegration rank is $r-1$

In practice, in determining the cointegration rank we first test the null of $r=m$, that is to say, apply a unit root test that considers the null of $I(0)$ for the first principal component. If the null hypothesis is accepted, the cointegration rank is decided to be $m$. If it is rejected, we test the hypothesis of $r=m-1$ or the degree of integration of the second principal component. We sequentially continue to test the null of $I(0)$ for the $m-r+1$ th principal component while the null hypothesis is rejected. When the 
null of $r=1$ is rejected, the cointegration rank is determined to be zero. In this testing procedure, we should apply a unit root test that considers the null of $I(0)$ for the $m-r$ th principal component, e.g., Kwiatkowski et al.'s (1992) test.

\section{MONTE CARLO SIMULATION}

In this section we examine the small sample properties of the test in the context of the following $m$-variate system:

$$
\Delta y_{t}=\alpha \beta^{+\prime} y_{t-1}^{+}+\varepsilon_{t}
$$

where $y_{t-1}^{+}=\left[1, y_{t-1}^{\prime}\right]^{\prime}, \beta^{+}=\left[\rho_{0}, \beta^{\prime}\right]^{\prime}, \alpha$ is an $(m \times r)$ matrix with rank $r$ and $\rho_{0}$ is an $(r \times 1)$ parameter vector. System $(7)$ is called the vector error correction model and derived from VAR(1). We compare our test to Johansen's by producing variations in the data generating process (7). We consider the following five variations:

Case 1. $m=2, r=1, \rho_{0}=0$ and $\varepsilon_{t} \sim$ i.i.d. $N\left(0, I_{2}\right)$. This is a simple system within Johansen's framework.

Case 2. $m=2, r=1, \rho_{0}=0.5$ and $\varepsilon_{t} \sim$ i.i.d. $N\left(0, I_{2}\right)$. There is a deterministic term in the cointegrating relation.

Case 3. $m=2, r=1, \rho_{0}=0, \varepsilon_{t}=0.5 u_{t-1}+u_{t}$ and $u_{t} \sim$ i.i.d.N $\left(0, I_{2}\right)$. In this case, since the disturbances follow a moving average process one of the assumptions of Johansen's tests, that is, the disturbances follow a Gaussian i.i.d. process, is violated.

Case 4. $m=5, r=3, \rho_{0}=0$ and $\varepsilon_{t} \sim$ i.i.d. $N\left(0, I_{5}\right)$. We consider a large cointegrated system.

Case 5. $m=15, r=10, \rho_{0}=0$ and $\varepsilon_{t} \sim$ i.i.d. $N\left(0, I_{15}\right)$. We consider an even larger cointegrated system than in Case 4. In this case, Johansen's tests are not feasible because the critical values are not available. 
For Cases 1, 2 and 3 we set

$$
\beta=\left[\begin{array}{l}
0.4 \\
0.1
\end{array}\right], \quad \alpha=\left[\begin{array}{c}
-1 \\
0
\end{array}\right] .
$$

Due to space limitations, we do not report $\beta$ and $\alpha$ for Cases 4 and 5 .

We choose the PP test with nominal size 0.01 for the unit root test for the principal component. The critical values for the PP test statistic come from COINT 2.0 which is the application module for GAUSS. Johansen's trace and $\lambda$-max test are implemented with a VAR(1) model except for Case 3. In Case 3, following Said and Dickey (1984), we approximate system (7) by VAR with order 3 for $T=100,4$ for $T=200$ and 5 for $T=400$ to apply Johansen's tests in an appropriate manner. The critical values of Johansen's tests statistic come from Osterwald-Lenum (1992). The nominal sizes for Johansen's tests are 0.01 . In each case, we use the sample sizes $T=100,200,400$. The number of replications is 10000 .

Case 1.

Table 1 (Empirical distribution of the selected cointegration rank in Case 1)

\begin{tabular}{lccc|ccc|ccc}
\hline & \multicolumn{3}{c|}{ Trace } & \multicolumn{3}{c|}{$\lambda$-max } & \multicolumn{3}{c}{ Our test } \\
\hline$T \backslash \hat{r}$ & 0 & 1 & 2 & 0 & 1 & 2 & 0 & 1 & 2 \\
\hline 100 & 1.4 & 97.4 & 1.3 & 1.3 & 97.4 & 1.3 & 0.2 & 98.8 & 1.0 \\
\hline 200 & 0.0 & 98.9 & 1.1 & 0.0 & 98.9 & 1.1 & 0.0 & 99.0 & 1.0 \\
\hline 400 & 0.0 & 98.6 & 1.4 & 0.0 & 98.6 & 1.4 & 0.0 & 98.9 & 1.1 \\
\hline
\end{tabular}

Notes: "Trace" indicates the trace test.

" $\lambda$-max" indicates the maximum eigenvalue test.

"Our test" indicates the proposed test.

$\hat{r}$ indicates the possible cointegration rank to be selected.

Table 1 shows the results of the experiment in Case 1. For trace and $\lambda$-max, the frequency of correct answers is $97.4 \%$ for $T=100$. On the other hand, the rate at which the proposed test finds the true rank of cointegration is $98.8 \%$ for $T=100$. Thus, the proposed test slightly outperforms Johansen's tests when the sample size is small. The frequency of finding the true rank increases for all tests for $T=200$ and 400 and their numerical performance is very similar. 
Case 2.

Table 2 (Empirical distribution of the selected cointegration rank in Case 2)

\begin{tabular}{cccc|ccc|ccc}
\hline & \multicolumn{3}{c|}{ Trace } & \multicolumn{3}{c|}{$\lambda$-max } & \multicolumn{3}{c}{ Our test } \\
\hline$T \backslash \hat{r}$ & 0 & 1 & 2 & 0 & 1 & 2 & 0 & 1 & 2 \\
\hline 100 & 12.2 & 87.0 & 0.8 & 8.6 & 90.6 & 0.8 & 1.2 & 97.8 & 1.0 \\
\hline 200 & 0.0 & 99.2 & 0.8 & 0.0 & 99.2 & 0.8 & 0.0 & 98.9 & 1.1 \\
\hline 400 & 0.0 & 99.1 & 0.9 & 0.0 & 99.1 & 0.9 & 0.0 & 99.1 & 0.9 \\
\hline
\end{tabular}

In Case 2, the performance of Johansen's tests depends on the sample size (see Table 2 ), with the test performing better the larger the sample. In contrast, the proposed test works properly even when the sample size is small.

Case 3.

Table 3 (Empirical distribution of the selected cointegration rank in Case 3)

\begin{tabular}{cccc|ccc|ccc}
\hline & \multicolumn{3}{c|}{ Trace } & \multicolumn{3}{c|}{$\lambda$-max } & \multicolumn{3}{c}{ Our test } \\
\hline$T \backslash \hat{r}$ & 0 & 1 & 2 & 0 & 1 & 2 & 0 & 1 & 2 \\
\hline 100 & 43.8 & 55.0 & 1.3 & 47.5 & 51.2 & 1.3 & 5.8 & 93.7 & 0.6 \\
\hline 200 & 0.5 & 98.1 & 1.4 & 0.6 & 98.0 & 1.4 & 0.0 & 99.5 & 0.5 \\
\hline 400 & 0.0 & 98.7 & 1.3 & 0.0 & 98.7 & 1.3 & 0.0 & 99.1 & 0.9 \\
\hline
\end{tabular}

Table 3 indicates that the MA(1) disturbances trigger a sharp drop in the frequency of correct answers of Johansen's tests, especially when $T=100$ despite an autoregressive approximation. In contrast, the proposed test performs reasonably well even when the sample size is only 100. All tests show a better performance with larger sample sizes but significant differences seem to occur in the performance of Johansen's tests and the proposed test for $T=200$. Johansen's tests need at least $T=400$ to work as well as the proposed test.

Case 4. 
Table 4 (Empirical distribution of the selected cointegration rank in Case 4)

\begin{tabular}{ccccccc|cccccc}
\hline \multicolumn{7}{c|}{ Trace } & \multicolumn{7}{c}{$\lambda$-max } \\
\hline$T \backslash \hat{r}$ & 0 & 1 & 2 & 3 & 4 & 5 & 0 & 1 & 2 & 3 & 4 & 5 \\
\hline 100 & 0.0 & 10.0 & 70.6 & 18.8 & 0.6 & 0.1 & 0.0 & 30.5 & 51.1 & 18.1 & 0.3 & 0.1 \\
\hline 200 & 0.0 & 0.0 & 7.8 & 91.2 & 1.0 & 0.0 & 0.0 & 0.0 & 3.5 & 95.7 & 0.7 & 0.0 \\
\hline 400 & 0.0 & 0.0 & 0.0 & 98.8 & 1.1 & 0.1 & 0.0 & 0.0 & 0.0 & 99.1 & 0.9 & 0.1 \\
\hline
\end{tabular}

\begin{tabular}{lcccccc}
\hline \multicolumn{7}{c}{ Our test } \\
\hline$T \backslash \hat{r}$ & 0 & 1 & 2 & 3 & 4 & 5 \\
\hline 100 & 0.0 & 1.0 & 37.4 & 61.3 & 0.3 & 0.0 \\
\hline 200 & 0.0 & 0.0 & 0.1 & 98.8 & 1.1 & 0.0 \\
\hline 400 & 0.0 & 0.0 & 0.0 & 98.8 & 1.2 & 0.0 \\
\hline
\end{tabular}

In a large cointegrated system, Johansen's tests exhibit severe distortions for $T=100$ (see Table 4). Johansen's tests need at least $T=200$ to work about as well as the proposed test. Particularly with a small sample, our test performs better than Johansen's.

Case 5.

Table 5 (Empirical distribution of the selected cointegration rank in Case 5)

\begin{tabular}{ccccccccccccccccc}
\hline \multicolumn{11}{c}{ Our test } \\
\hline$T \backslash \hat{r}$ & 0 & 1 & 2 & 3 & 4 & 5 & 6 & 7 & 8 & 9 & 10 & 11 & 12 & 13 & 14 & 15 \\
\hline 100 & 0.0 & 0.1 & 0.2 & 0.4 & 2.0 & 6.5 & 16.0 & 30.1 & 29.5 & 13.2 & 2.0 & 0.1 & 0.0 & 0.0 & 0.0 & 0.0 \\
\hline 200 & 0.0 & 0.0 & 0.0 & 0.0 & 0.1 & 0.2 & 1.6 & 6.4 & 18.5 & 36.0 & 35.1 & 2.1 & 0.0 & 0.0 & 0.0 & 0.0 \\
\hline 400 & 0.0 & 0.0 & 0.0 & 0.0 & 0.0 & 0.0 & 0.0 & 0.0 & 0.0 & 0.3 & 93.6 & 5.9 & 0.1 & 0.0 & 0.0 & 0.0 \\
\hline 1000 & 0.0 & 0.0 & 0.0 & 0.0 & 0.0 & 0.0 & 0.0 & 0.0 & 0.0 & 0.0 & 95.0 & 5.0 & 0.1 & 0.0 & 0.0 & 0.0 \\
\hline
\end{tabular}

For Case 5 we only report the result of our test since Johansen's tests cannot be used due to the lack of critical values. For $T=100$, our test has the cointegration rank right in only $2.0 \%$ of the cases (see Table 5). However, with a sample size of 400, the performance increases substantially, with the test correctly detecting the cointegration rank in $93.6 \%$ of the cases. This indicates that our test requires a larger sample size to perform reasonably well in the case of a larger cointegrated system. We therefore also examine the properties of the test for $T=1000$ and find that although the proportion of correct answers increases to $95.0 \%$, the rate of correct answers seems to rise only slowly in a larger cointegrated system. 


\section{Summary of the experiments.}

The experiments indicate that the proposed test performs slightly better than Johansen's tests even in a simple model within Johansen's framework with a small sample size (Case 1). A deterministic term or large $m$ sharpen the contrast between the proposed test and Johansen's for a small $T$ (Case 2 and Case 4). If a model does not satisfies the assumptions for Johansen's tests (Case 3) or becomes so large that Johansen's tests are not applicable (Case 5), our test performs well. The results reported in this paper are representative of all the experiments we performed, which similarly indicated that our test is generally superior to Johansen's tests. However, we should note that there can be cases where the proposed test is inferior to Johansen's tests, especially in Case 1, depending upon parameter values of DGP. In Cases 2, 3 and 4, there also can be cases where the test has no advantage over Johansen's tests, although this is quite rare. Our test therefore is preferable except in quite simple cases. Because of space limitations, we cannot report here all of the experiments that we conducted. The results are available on request.

\section{CONCLUDING REMARKS}

In this paper, we proposed a new cointegration rank test. The test has some attractive features. Because the testing procedure consists of a unit root test on each principal component, applying an appropriate unit root test allows the test to be less restrictive than Johansen's. For example, applying the PP test, the proposed test becomes less restrictive than Johansen's in the sense that it does not require normally distributed disturbances and VAR representation of the systems. Applying a commonly used unit root test such as the PP test, one can compute the test statistic easily and utilize widelyacknowledged critical values. The test is independent of the dimension of the time series. This property is useful for a cointegration test in large systems where the critical values for existing cointegration tests are not practically available.

We employed the Monte Carlo method to compare the performance of tests of coin- 
tegration. The cointegration rank is determined by the trace test, the $\lambda$-max test and the proposed test and the distribution of the determined cointegration rank is compared. Although Johansen's tests perform as well as the proposed test when the sample size is sufficiently large, the proposed test is superior in terms of the frequency of correct answers when the sample size is small. 


\section{References}

Breitung, J. (2002): "Nonparametric tests for unit roots and cointegration," Journal of Econometrics, 108, 343-363.

Harris, D. (1997): "Principal components analysis of cointegrated time series," Econometric Theory, 13, 529-557.

Hubrich, K., H. Leutkepohl and P. Saikkonen (2001): "A review of systems cointegration Tests," Econometric Reviews,, 20, 247-318.

Johansen, S. (1988): "Statistical analysis of cointegration vectors," Journal of Economic Dynamics and Control, 12, 231-254.

Johansen, S. (1991): "Estimation and hypothesis testing of cointegration vectors in Gaussian vector autoregressive models," Econometrica, 59, 1551-1580.

Kwiatkowski, D., P.C.B. Phillips, P. Schmidt and Y. Shin (1992): "Testing the null hypothesis of stationarity against the alternative of a unit root," Journal of Econometrics, 54, 159-178.

Lucas, A. (1997): "Cointegration testing using pseudo likelihood ratio tests," Econometric Theory, 13, 149-169.

Lucas, A. (1998): "Inference on cointegrating ranks using LR and LM tests based on pseudolikelihoods," Econometric Reviews, 17, 185-214.

Phillips, P.C.B. (1987): "Time series regression with a unit root," Econometrica, 55, $277-301$.

Phillips, P.C.B. (1995): "Fully modified least squares and vector autoregression," Econometrica, 63, 1023-1078.

Phillips, P.C.B. and P. Perron (1988): "Testing for a unit root in time series regression," Biometrika, 75, 335-346. 
Quintos, C.E. (1998): "Fully modified vector autoregressive inference in partially nonstationary models.," Journal of the American Statistical Association, 93, 783-795.

Said, S.E. and D.A. Dickey (1984): "Testing for unit roots in autoregressive-moving average models of unknown order," Biometrika, 71, 599-607.

Snell, A. (1999): "Testing for $r$ versus $r-1$ cointegrating vectors," Journal of Econometrics, 88, 151-191

Stock, J.H. and M. Watson (1988): "Testing for common trends," Journal of the American Statistical Association, 83, 1097-1107. 
Appendix Proof of Proposition 2

To prove Proposition 2(i), we express $(1-L) \bar{x}_{i}$ as follows:

$$
\begin{aligned}
(1-L) \bar{x}_{i} & =\left(\beta_{\perp}\left(\beta_{\perp}^{\prime} \beta_{\perp}\right)^{-1} \beta_{\perp}^{\prime}+\beta\left(\beta^{\prime} \beta\right)^{-1} \beta^{\prime}\right)(1-L) \bar{x}_{i} \\
& =\beta_{\perp}\left(\beta_{\perp}^{\prime} \beta_{\perp}\right)^{-1} \times \beta_{\perp}^{\prime}(1-L) \bar{x}_{i}+\beta\left(\beta^{\prime} \beta\right)^{-1} \times \beta^{\prime}(1-L) \bar{x}_{i} \\
& \left.=\beta_{\perp}\left(\beta_{\perp}^{\prime} \beta_{\perp}\right)^{-1} w_{i}+\beta\left(\beta^{\prime} \beta\right)^{-1}(1-L) z_{i} . \quad \text { (say }\right)
\end{aligned}
$$

Summing from $i=1, \cdots, t$ we get

$$
\bar{x}_{t}=\beta_{\perp}\left(\beta_{\perp}^{\prime} \beta_{\perp}\right)^{-1} \sum_{i=1}^{t} w_{i}+\beta\left(\beta^{\prime} \beta\right)^{-1} z_{t}+\bar{x}_{0}-\beta\left(\beta^{\prime} \beta\right)^{-1} z_{0} .
$$

After multiplying equation (8) by $B_{(m-r)}^{\prime}$, we apply (5) to derive the following equation:

$$
\begin{aligned}
B_{(m-r)}^{\prime} \bar{x}_{t}= & \left(\beta_{\perp}^{\prime} B_{(m-r)}\right)^{\prime}\left(\beta_{\perp}^{\prime} \beta_{\perp}\right)^{\prime-1} \beta_{\perp}^{\prime} \beta_{\perp}\left(\beta_{\perp}^{\prime} \beta_{\perp}\right)^{-1} \sum_{i=1}^{t} w_{i} \\
& +\left(\beta_{\perp}^{\prime} B_{(m-r)}\right)^{\prime}\left(\beta_{\perp}^{\prime} \beta_{\perp}\right)^{\prime-1} \beta_{\perp}^{\prime} \beta\left(\beta^{\prime} \beta\right)^{-1} z_{t} \\
& +\left(\beta_{\perp}^{\prime} B_{(m-r)}\right)^{\prime}\left(\beta_{\perp}^{\prime} \beta_{\perp}\right)^{\prime-1} \beta_{\perp}^{\prime}\left(\bar{x}_{0}-\beta\left(\beta^{\prime} \beta\right)^{-1} z_{0}\right)+O_{p}\left(T^{-1 / 2}\right) \\
= & \left(\beta_{\perp}^{\prime} B_{(m-r)}\right)^{\prime}\left(\beta_{\perp}^{\prime} \beta_{\perp}\right)^{\prime-1} \sum_{i=1}^{t} w_{i}+\left(\beta_{\perp}^{\prime} B_{(m-r)}\right)^{\prime}\left(\beta_{\perp}^{\prime} \beta_{\perp}\right)^{\prime-1} \beta_{\perp}^{\prime}\left(\bar{x}_{0}-\beta\left(\beta^{\prime} \beta\right)^{-1} z_{0}\right) \\
& +O_{p}\left(T^{-1 / 2}\right) \\
\approx & Q^{\prime} \sum_{i=1}^{t} w_{i}+Q^{\prime} \beta_{\perp}^{\prime}\left(\bar{x}_{0}-\beta\left(\beta^{\prime} \beta\right)^{-1} z_{0}\right) .
\end{aligned}
$$

The approximation (9) holds as $T$ gets large. Because $Q$ is a matrix of full rank as mentioned in Remark 2, each component of $B_{(m-r)}^{\prime} \bar{x}_{t}$ is $I(1)$ asymptotically. This completes the proof of Proposition 3.2(i).

Next, we prove Proposition 2(ii). After multiplying equation (8) by $B_{(r)}^{\prime}$, we apply (6) 
to derive the following equation:

$$
\begin{aligned}
B_{(r)}^{\prime} \bar{x}_{t}= & \left(\beta^{\prime} B_{(r)}\right)^{\prime}\left(\beta^{\prime} \beta\right)^{\prime-1} \beta^{\prime} \beta_{\perp}\left(\beta_{\perp}^{\prime} \beta_{\perp}\right)^{-1} \sum_{i=1}^{t} w_{i}+\left(\beta^{\prime} B_{(r)}\right)^{\prime}\left(\beta^{\prime} \beta\right)^{\prime-1} \beta^{\prime} \beta\left(\beta^{\prime} \beta\right)^{-1} z_{t} \\
& +\left(\beta^{\prime} B_{(r)}\right)^{\prime}\left(\beta^{\prime} \beta\right)^{\prime-1} \beta^{\prime}\left(\bar{x}_{0}-\beta\left(\beta^{\prime} \beta\right)^{-1} z_{0}\right)+O_{p}\left(T^{-1 / 2}\right) \\
= & \left(\beta^{\prime} B_{(r)}\right)^{\prime}\left(\beta^{\prime} \beta\right)^{\prime-1} z_{t}+\left(\beta^{\prime} B_{(r)}\right)^{\prime}\left(\beta^{\prime} \beta\right)^{\prime-1} \beta^{\prime}\left(\bar{x}_{0}-\beta\left(\beta^{\prime} \beta\right)^{-1} z_{0}\right)+O_{p}\left(T^{-1 / 2}\right) \\
\approx & R^{\prime} z_{t}+R^{\prime} \beta^{\prime}\left(\bar{x}_{0}-\beta\left(\beta^{\prime} \beta\right)^{-1} z_{0}\right) .
\end{aligned}
$$

This completes the proof of Proposition 2(ii). 